\title{
Adsorção de azul de metileno em hidrocarvões de resíduos têxteis
}

\author{
Methylene blue adsorption in hydrocarbons \\ textile waste
}

\author{
Adriana Ferreira de Lima ${ }^{1}$, Helida Monique Cordasso Fagnani ${ }^{1,2}$, \\ Washington Luiz Félix Santos ${ }^{1}$, Maria Angélica Simões Dornellas de Barros ${ }^{1}$
}

\footnotetext{
${ }^{1}$ Universidade Estadual de Maringá, UEM, Av. Colombo, 5790 - Zona 7, CEP: 87020-900, Maringá, PR, Brasil.

${ }^{2}$ Instituto Federal de Educação Ciência e Tecnologia do Tocantins, IFTO, 310 Sul, Av. LO 5, s/n, Plano Diretor Sul, CEP: 77021-090 Palmas, TO, Brasil.

e-mail: adrianashynoda@hotmail.com, helidamonique@gmail.com,wlfsantos@uem.br, angelicabarros.deq@gmail.com
}

\section{RESUMO}

Os produtos têxteis, cada vez mais presentes em nosso dia-a-dia, geram uma grande quantidade de resíduos, sejam eles de forma sólida ou líquida. Dar o destino correto ou até mesmo transformar os resíduos sólidos é o novo desafio a ser enfrentado. Os resíduos sólidos, geralmente retalhos de tecidos, contém grande quantidade de compostos orgânicos, que podem ser carbonizados. Neste contexto, o principal objetivo do presente estudo foi a síntese de hidrocarvão utilizando resíduos têxteis. Os hidrocarvões formados podem ser utilizados como adsorventes de corantes, como o azul de metileno. A síntese de hidrocarvões utilizou uma razão resíduo/água de 3/80 massa $(\mathrm{g}) /$ volume $(\mathrm{mL})$ a uma temperatura de $220^{\circ} \mathrm{C}$. O tempo foi investigado entre 6 e 12 horas, sendo que este último obteve $71,2 \%$ de carbono, obtido por EDS e seguiu para caracterização por FTIR-ATR, DRX, MEV e $\mathrm{pH}_{\mathrm{pcz}}$. $\mathrm{O}$ material proposto apresentou significativa capacidade de adsorção em meio básico, em torno de $72 \mathrm{mg} \cdot \mathrm{g}^{-1}$. Os dados cinéticos obtidos foram melhor ajustados no modelo de pseudo segunda-ordem sugerindo que o processo de adsorção foi controlado por quimissorção, ocorrendo o compartilhamento ou troca de elétrons entre adsorvente e adsorvato. O modelo de Langmuir apresentou melhor correlação com os dados de equilíbrio. Os hidrocarvões sintetizados com resíduos têxteis apresentam, portanto, grande potencial em aplicações, visando à remoção de poluentes orgânicos de solução aquosa.

Palavras-Chave: carbonização hidrotérmica; resíduos têxteis; adsorção de azul de metileno.

\begin{abstract}
Textile products, increasingly present in our daily lives, generate a large amount of waste, whether solid or liquid. Giving the right destination or even transforming solid waste is the new challenge to be faced. Solid waste, usually tissue scraps, contains a large amount of organic compounds that can be carbonized. In this context, the main objective of the present study was the synthesis of hydrochars using textile waste. The hydrochars formed may be used as dye adsorbents such as methylene blue. Hydrochars synthesis used a residue/water ratio of $3 / 80$ mass $(\mathrm{g}) /$ volume $(\mathrm{mL})$ at a temperature of $220^{\circ} \mathrm{C}$. The time was investigated between 6 to 12 hours, and in the latter there was $71.2 \%$ carbon, obtained by EDS, and followed for characterization by ATR-FTIR, XDR, SEM and $\mathrm{pH}_{\mathrm{zpc}}$. The proposed material presented significant adsorption capacity in alkaline environment, around $72 \mathrm{mg} \cdot \mathrm{g}^{-1}$. The kinetic data obtained were better adjusted in the pseudo secondorder model suggesting that the adsorption process was controlled by chemissorption, with electron sharing or exchange between adsorbent and adsorbate. Langmuir model presented better correlation with equilibrium data. Hydrochars synthesized with textile waste therefore have great potential in applications, aiming at the removal of organic pollutants from liquid solutions.
\end{abstract}

Keywords: hydrothermal carbonization; textile waste; methylene blue adsorption. 


\section{INTRODUÇÃO}

O início do século XXI apresenta diversos desafios. Um deles se refere ao desenvolvimento de tecnologias que promovam conforto e bem-estar à população, e que mantenham o compromisso de sustentabilidade. Considerada como o segundo maior empregador da indústria de transformação, a indústria têxtil situa-se como mantenedora de grande escala produtiva. Consequentemente, emerge como uma das grandes responsáveis na geração de resíduos nocivos ao ambiente, enfrentando assim enormes desafios ambientais e de recurso [1].

O processo de desenvolvimento de produtos de vestuário produz uma grande quantidade de resíduos, principalmente quanto ao setor de corte nas confecções, em que toneladas de retalhos são, muitas vezes, descartados de modo displicente em aterros sanitários gerando um enorme passivo ambiental [2,1].

A opção que deveria ser seguida pelas indústrias é a reciclagem ou a reutilização dos materiais descartados em outros processos resultando em outros produtos, de maior valor agregado [2]. Felizmente hoje há empresas de reciclagem que transformam resíduos têxteis em novos produtos, como, por exemplo, transforma os resíduos têxteis em fibras regeneradas, formando um novo fio, que em processo de desfibração, podem ser produzidos tecidos planos ou de malhas [2], entretanto, ainda há uma grande quantidade de resíduos a serem descartados. Uma possibilidade é a utilização destes resíduos têxteis como matéria-prima para a produção de hidrocarvão sem ativação, para fins de utilização como adsorvente.

A técnica mais conhecida para a formação de carvão é a pirólise, que consiste em submeter o resíduo a altas temperaturas $\left(600-900^{\circ} \mathrm{C}\right)$ na ausência de oxigênio, ocorrendo à ruptura da estrutura molecular original [3]. Porém, nos últimos anos, uma atenção considerável tem sido dada para a produção de hidrocarvões a partir da carbonização hidrotérmica (CHT) de biomassa de resíduos úmidos, tais como biomassa de resíduos [4,5], resíduos sólidos urbanos [6,7], lodo [8,9], etc. Entre as vantagens, em relação à pirólise convencional estão a baixa temperatura de síntese e o fato de ser um processo ambientalmente amigável, devido ao uso somente de água no processo [10].

Inúmeros trabalhos demonstram que a CHT é um processo ambientalmente vantajoso para o tratamento de resíduos ou biomassas, e o carvão assim obtido é similar aos carvões obtidos por pirólise [11-17].

Neste cenário, a síntese de hidrocarvões de rejeitos têxteis auxilia na diminuição de resíduos sólidos e o hidrocarvão formado pode ser utilizado em processos de adsorção para tratamento de efluentes líquidos do próprio setor têxtil.

Um exemplo dessa possível aplicação está relacionado à remoção de corantes que são alguns dos principais componentes das águas residuais de origem industrial, principalmente do setor da indústria têxtil. Devido aos efeitos deletérios causados, seja pelo escurecimento da água, seja pela própria contaminação com moléculas que não fazem parte do ecossistema, a remoção de corantes de efluentes é um dos requisitos mais difíceis no tratamento [18]. O azul de metileno é um corante frequentemente investigado, devido a sua forte adsorção em sólidos, servindo como um composto modelo para estudo de adsorção de contaminantes orgânicos em soluções aquosas e em sua estrutura molecular percebe-se o caráter catiônico do grupo amino, favorecendo maiores interações eletrostáticas em pH básico [19].

Assim sendo, o objetivo deste trabalho foi investigar o tempo de hidrocarbonização de resíduos sólidos têxteis e sua utilização como adsorvente de um dos mais conhecidos compostos modelo para contaminantes orgânicos.

\section{MATERIAIS E MÉTODOS}

\section{$1.1 \quad$ Materiais}

Os resíduos têxteis foram cedidos por uma indústria de confecção, situada na cidade de Cianorte-PR. Os resíduos gerados no setor de corte da empresa, continham tecidos de brim alvejado com mistura de algodão e elastano em sua composição.

$\mathrm{O}$ corante catiônico azul de metileno Vertec ${ }^{\circledR}$, de fórmula molecular $\mathrm{C}_{16} \mathrm{H}_{18} \mathrm{C}_{1} \mathrm{~N}_{3} \mathrm{~S}$ foi utilizado nos experimentos. Suas dimensões moleculares são 14,3 $\AA$ de largura, 6,1 $\mathrm{A}$ de profundidade, $4 \AA$ de espessura, com um diâmetro molecular de $8 \AA$ e volume molecular de $241,9 \mathrm{~cm}^{3} \cdot \mathrm{mol}^{-1}$ [20]. As soluções foram preparadas pela dissolução do corante em água destilada nas concentrações de 10 a $300 \mathrm{mg} \cdot \mathrm{L}^{-1}$, conforme as seções 2.3.1, 2.3.2 e 2.3.3. A análise da concentração de azul de metileno em solução foi realizada no espectrofotômetro UV-Vis da Shimadzu modelo UV-1800, no comprimento de onda $\left(\lambda_{\max }\right)$ de $664 \mathrm{~nm}$ [23]. 


\subsection{Síntese do hidrocarvão de resíduos têxteis}

Os experimentos de CHT foram realizados utilizando-se um reator de aço de inox revestido internamente com uma camada de teflon de capacidade de $120 \mathrm{~mL}$. No reator foram adicionados $3,0 \mathrm{~g}$ de retalhos e $80 \mathrm{~mL}$ de água destilada. Tal sistema foi submetido à carbonização com duração de 6, 8, 10 ou 12 horas a $220^{\circ} \mathrm{C}$ [24]. Logo após o referido processo, os hidrocarvões resultantes foram secos em estufa por 5 horas a $105^{\circ} \mathrm{C}$. Os experimentos foram realizados em quintuplicata.

Por fim o hidrocarvão foi pesado e denominado com a inicial HC, seguido do tempo de hidrocarbonização a saber 6, 8, 10 ou 12 horas. O rendimento em hidrocarvão (RH) foi determinado pela equação1.

$$
R H(\%)=\frac{\text { Peso do hidrocarvão }}{\text { Peso da matéria-prima }} X 100
$$

Os resíduos têxteis foram caracterizados pelas normas NBR 13538:1995[21], NBR 11914:1992[22], no Instituto de Pesos e Medidas do Estado do Paraná (IPEM-PR), de Londrina-PR. Tal análise consiste em determinar a composição do material, qualitativamente e quantitativamente.

A morfologia do resíduo têxtil bruto e dos hidrocarvões foi analisada por microscopia eletrônica de varredura (MEV) com energia dispersiva de raio X (EDS) (FEI company, modelo Quanta -250). A espectroscopia no infravermelho por refletância total atenuada (FTIR-ATR) foi realizada usando espectrofotômetro Shimadzu, modelo IRTracer-100 com cristal de germânio, no intervalo de 4000 a $400 \mathrm{~cm}^{-1}$ com resolução de $4 \mathrm{~cm}^{-1}$ e 45 scans. As estruturas cristalinas, no caso do carvão a estrutura amorfa, das amostras foram analisadas por difração raio $X(D R X)$ com aquisição automática de dados, a $30 \mathrm{~V}$ e $10 \mathrm{~mA}$, comprimento de onda de $1,54 \AA$, emitida por um núcleo de cobre. As amostras foram analisadas em ângulos na faixa de $10-80^{\circ}(2 \theta)$. O ponto de carga zero $\left(\mathrm{pH}_{\mathrm{pcz}}\right)$ foi determinado usando o método proposto por Park e Regabulto [25], somente na amostra selecionada como possível adsorvente.

\subsection{Ensaios de Adsorção}

O hidrocarvão que obteve maior quantidade de carbono foi utilizado para a adsorção do corante azul de metileno. Os ensaios de adsorção foram realizados utilizando-se $10 \mathrm{mg}$ do adsorvente e $20 \mathrm{~mL}$ de solução de azul de metileno com concentrações variadas. A temperatura de cada teste foi de $25^{\circ} \mathrm{C}$, em agitação constante em um equipamento de tingimento Kimak AT1-SW com rotação de $70 \mathrm{rpm}$. A quantidade de azul de metileno adsorvido em $\mathrm{mg}^{-1} \mathrm{~g}^{-1}$ foi calculada pelo balanço de massa, conforme a equação 2 :

$$
q=\frac{\left(C_{0}-C\right) \cdot V}{M}
$$

em que $\mathrm{C}_{0}$ é a concentração inicial do azul de metileno; $\mathrm{C}$ é a concentração de azul de metileno na solução ao fim de cada teste; V é o volume de solução utilizada $(20 \mathrm{~mL})$; M é a massa seca de hidrocarvão; e q é a quantidade adsorvida ao final de cada teste.

\subsubsection{Influência do pH}

A influência do pH foi avaliada variando o pH de 3,0 a 10,0, utilizando $\mathrm{NaOH}$ ou $\mathrm{HCl}\left(0,1 \mathrm{~mol}^{-1} \mathrm{~L}^{-1}\right)$. Em cada caneca foi adicionado $10 \mathrm{mg}$ de hidrocarvão e $20 \mathrm{~mL}$ da solução azul de metileno $\left(50 \mathrm{mg} . \mathrm{L}^{-1}\right)$ por 24 horas com os respectivos valores de $\mathrm{pH}$. A quantidade adsorvida de azul de metileno também foi calculada pelo balanço de massa, conforme a equação 2 . 


\subsubsection{Cinética de Adsorção}

O estudo cinético de adsorção foi realizado ao longo de 120 min com uma solução de 50 mg.L $\mathrm{L}^{-1}$ de azul de metileno no $\mathrm{pH}$ determinado pelo ensaio anterior. Os dados experimentais foram ajustados para os modelos cinéticos de pseudo-primeira ordem (Equação 3), pseudo-segunda ordem (Equação 4) e difusão intrapartícula (Equação 5) [26-28].

$$
\begin{aligned}
& \mathrm{q}_{\mathrm{t}}=\mathrm{q}_{\mathrm{eq}}\left(1-\mathrm{e}^{-\mathrm{k}_{1} \mathrm{t}}\right) \\
& \mathrm{q}_{\mathrm{t}}=\frac{\mathrm{t} \cdot \mathrm{q}_{\mathrm{eq}}{ }^{2} \cdot \mathrm{k}_{2}}{1+\mathrm{t} \cdot \mathrm{k}_{2} \cdot \mathrm{q}_{\mathrm{eq}}} \\
& q_{t}=k_{3} \cdot t^{0,5}+C
\end{aligned}
$$

em que: $\mathrm{k}_{1} ; \mathrm{k}_{2} ; \mathrm{k}_{3}$ são as velocidades específicas de adsorção do modelo de pseudo-primeira ordem, pseudosegunda ordem e difusão intrapartícula respectivamente, $\mathrm{q}_{\mathrm{eq}}$ é a quantidade adsorvida no equilíbrio e $\mathrm{q}_{\mathrm{t}}$ é a quantidade adsorvida no tempo t o parâmetro $\mathrm{C}$ da difusão intrapartícula é a constante do modelo que representa o efeito da camada limite.

\subsubsection{Isotermas de Adsorção}

As isotermas de adsorção foram obtidas a partir de soluções com concentrações de 10 a $300 \mathrm{mg} . \mathrm{L}^{-1}$, no pH determinado pelo teste de efeito de $\mathrm{pH}$ da solução sob agitação, até o tempo de equilíbrio. Os resultados experimentais foram correlacionados com os modelos de isotermas de Langmuir (Equação 6) [29], Freundlich (Equação 7) [30] e Sips (Equação 8) [31].

$$
\begin{aligned}
\mathrm{q}_{\mathrm{eq}} & =\frac{\mathrm{q}_{\mathrm{max}} \cdot \mathrm{b} \cdot \mathrm{C}_{\mathrm{eq}}}{1+\mathrm{b} \cdot \mathrm{C}} \\
\mathrm{q}_{\mathrm{eq}} & =\mathrm{K} \cdot \mathrm{C}_{\mathrm{eq}}^{1 / \mathrm{n}} \\
\mathrm{q}_{\mathrm{eq}} & =\frac{\mathrm{q}_{\max }\left(\mathrm{b} \cdot \mathrm{C}_{\mathrm{eq}}\right)^{1 / \mathrm{n}}}{1+\left(\mathrm{b} \cdot \mathrm{C}_{\mathrm{eq}}\right)^{1 / \mathrm{n}}}
\end{aligned}
$$

em que para Equação 6: $q_{\mathrm{eq}}$ é a quantidade de íons adsorvido por massa de adsorvente quando o sistema está em equilíbrio; $C_{\mathrm{eq}}$ é a concentração de equilíbrio do adsorvato na solução aquosa; b representa a razão entre a taxa de adsorção e dessorção; q máx indica a capacidade máxima de adsorvente. Para a Equação 7, k e n são constantes, se relacionam com a distribuição dos sítios ativos e a capacidade de adsorção do adsorvente.

Na Equação 8 o parâmetro 1/n é equivalente ao do modelo de Freundlich, que representa a heterogeneidade do sólido.

\section{RESULTADOS E DISCUSSÕES}

\subsection{Caracterizações do Resíduo Têxtil de Brim Alvejado e seus Hidrocarvões}

O resíduo têxtil, de acordo com as normas NBR 13538:1995[21] e NBR 11914:1992[22], apresentou os dados que seguem na Tabela 1. A partir dos resultados é possível avaliar que a amostra é composta praticamente por algodão, tecido bastante rico em carbono o que de fato, auxilia na hidrocarbonização. 
Tabela 1: Dados da composição do resíduo têxtil bruto.

\begin{tabular}{l|c|c}
\hline COMPOSIÇÃo & PERCENTAGEM (\%) & INCERTEZA (\%) \\
\hline Algodão & 96,59 & 1,52 \\
\hline Elastano & 3,41 & 1,52 \\
\hline
\end{tabular}

Na Tabela 2 são apresentadas as imagens fotográficas dos hidrocarvões, em tempos de CHT de 6, 8, 10 e 12 horas e seus respectivos rendimentos.

Tabela 2: Foto dos hidrocarvões produzidos do resíduo têxtil relacionados por temperatura, tempo de CHT e rendimento

\begin{tabular}{c|c|c|c|c|c}
\hline Temp. & $\begin{array}{c}\text { Resíduo Têxtil } \\
\text { Bruto }\end{array}$ & $6 \mathrm{~h}$ & $\mathbf{8 h}$ & $\mathbf{1 0 h}$ & $\mathbf{1 2 h}$ \\
\hline $\mathbf{2 2 0} \circ \mathrm{C}$ & & & & & \\
\hline
\end{tabular}

Por meio da Tabela 2 observa-se que o maior tempo de carbonização altera significativamente a cor do material resultante, para mais escuro. Além disso, a uniformidade do carvão também foi observada. $\mathrm{O}$ tempo de 6 horas não foi suficiente para carbonizar por completo o resíduo têxtil bruto, pois, visivelmente ainda apresenta um aspecto de tecido. As amostras de 8 e 10 horas foram carbonizadas, porém ainda possuem regiões mais claras, e alguns indícios de tecidos quando comparada com a de 12 horas. Além disso, este ensaio promoveu um material bastante fino.

Ainda na Tabela 2 observa-se uma diminuição significativa em peso do hidrocarvão em relação à matéria-prima com o tempo de hidrocarbonização, tal fato já havia sido reportado [32-37, 5, 17]. A perda de peso está relacionada com à perda de compostos como oxigênio e hidrogênio, aumentando, assim, a concentração de carbono na amostra final [35].

As microscopias eletrônicas de varredura foram obtidas para avaliar as morfologias do resíduo têxtil e dos seus hidrocarvões. As imagens em diferentes ampliações são mostradas na Figura 1.

O resíduo têxtil é formado por estrutura sem nenhum poro apresentando as fibras todas entrelaçadas. Somente a partir de 8 horas de CHT, há o surgimento de microesferas na superfície do hidrocarvão. As microesferas de carbono são resultantes da síntese controlada em temperatura baixa [38], podendo estar associada também à decomposição, condensação, desidratação ou polimerização e aromatização de componentes celulósicos durante a carbonização hidrotérmica [39, 40]. 

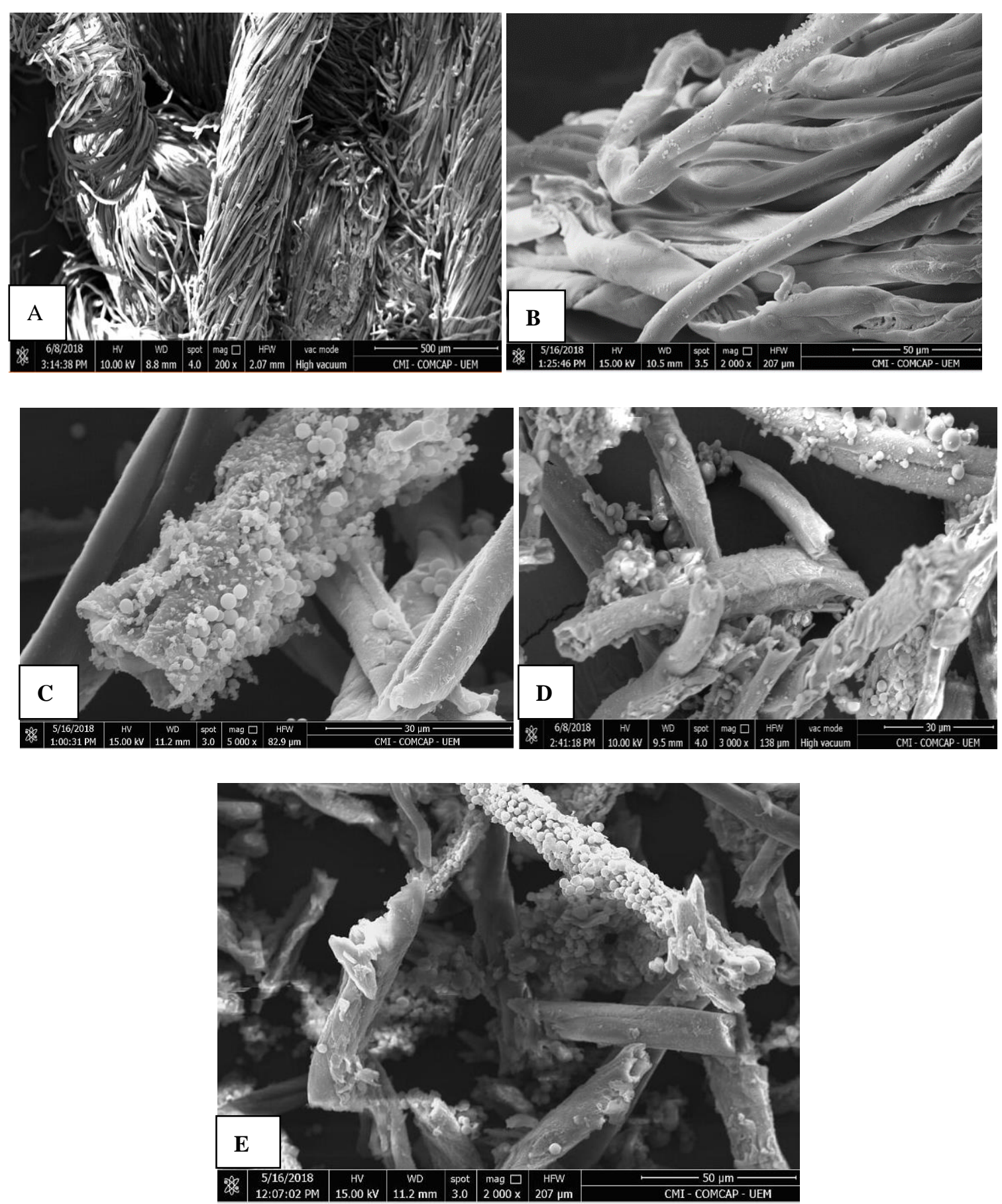

Figura 1: Microscopia eletrônica de varredura do resíduo têxtil bruto e hidrocarvões (a) resíduo têxtil, (b) hidrocarvão 6 h, (c) hidrocarvão 8 h, (d) hidrocarvão 10 h (e) hidrocarvão 12 h.

Na Figura 2 está exposta a análise de EDS com elementos e a porcentagem de cada um presente na amostra. Observa-se o aumento crescente de carbono e a diminuição gradativa de outros elementos com o tempo de carbonização, o que vem ao encontro das discussões anteriores. Na amostra de resíduo têxtil, este estudo encontrou $51,2 \%$ e $48,7 \%$ para o carbono e oxigênio respectivamente, valores estes semelhantes ao encontrado por Lin et al. [6], para hidrocarbonização também de resíduo têxtil. Já para o HC12, este trabalho encontrou $71,2 \%$ de carbono e $28,7 \%$ de oxigênio, enquanto Lin et al. [6] obtiveram 60,15\% de carbono e 34,41 de oxigênio, ou seja, resultados próximos aos encontrados nesse trabalho. O aumento dos rendimentos de carbono foi atribuído à redução da matéria volátil. 


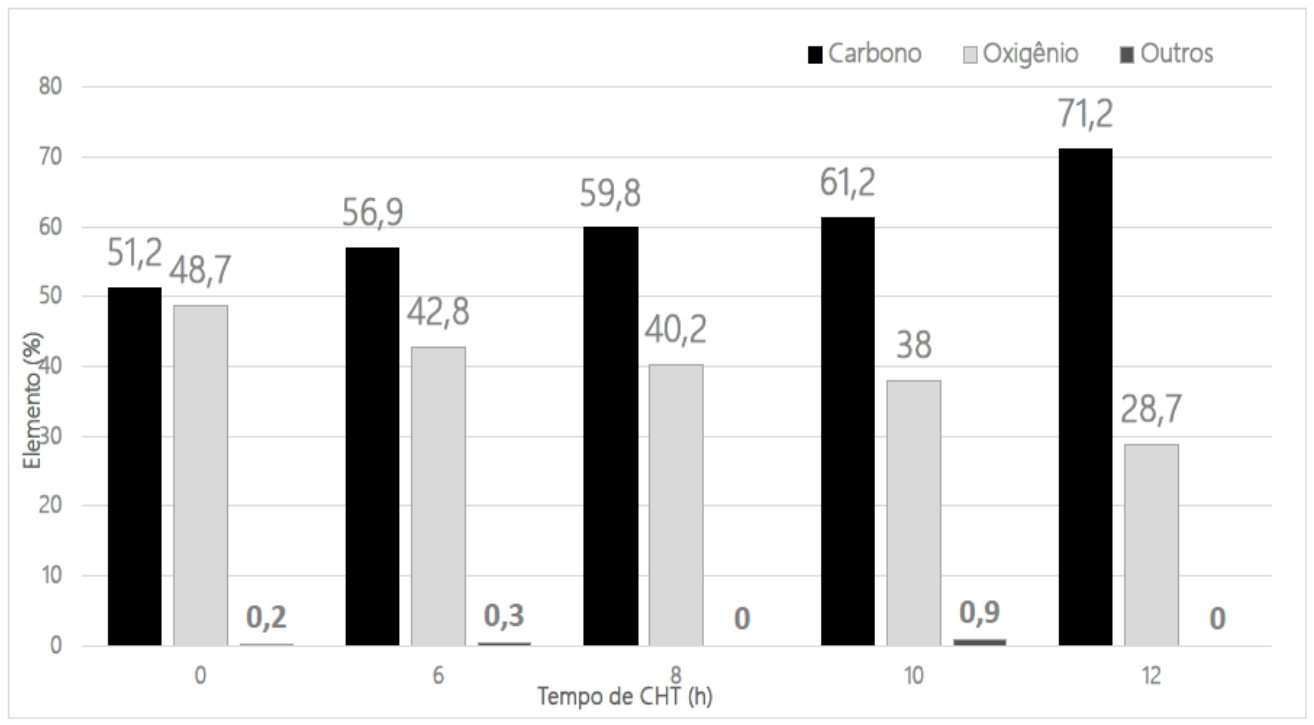

Figura 2: Análise de espectroscopia de dispersão de energia de raio X do resíduo têxtil bruto e dos hidrocarvões.

O FTIR-ATR das amostras do resíduo têxtil bruto e dos hidrocarvões de 6, 8, 10 e 12 horas de CHT está apresentado na Figura 3.

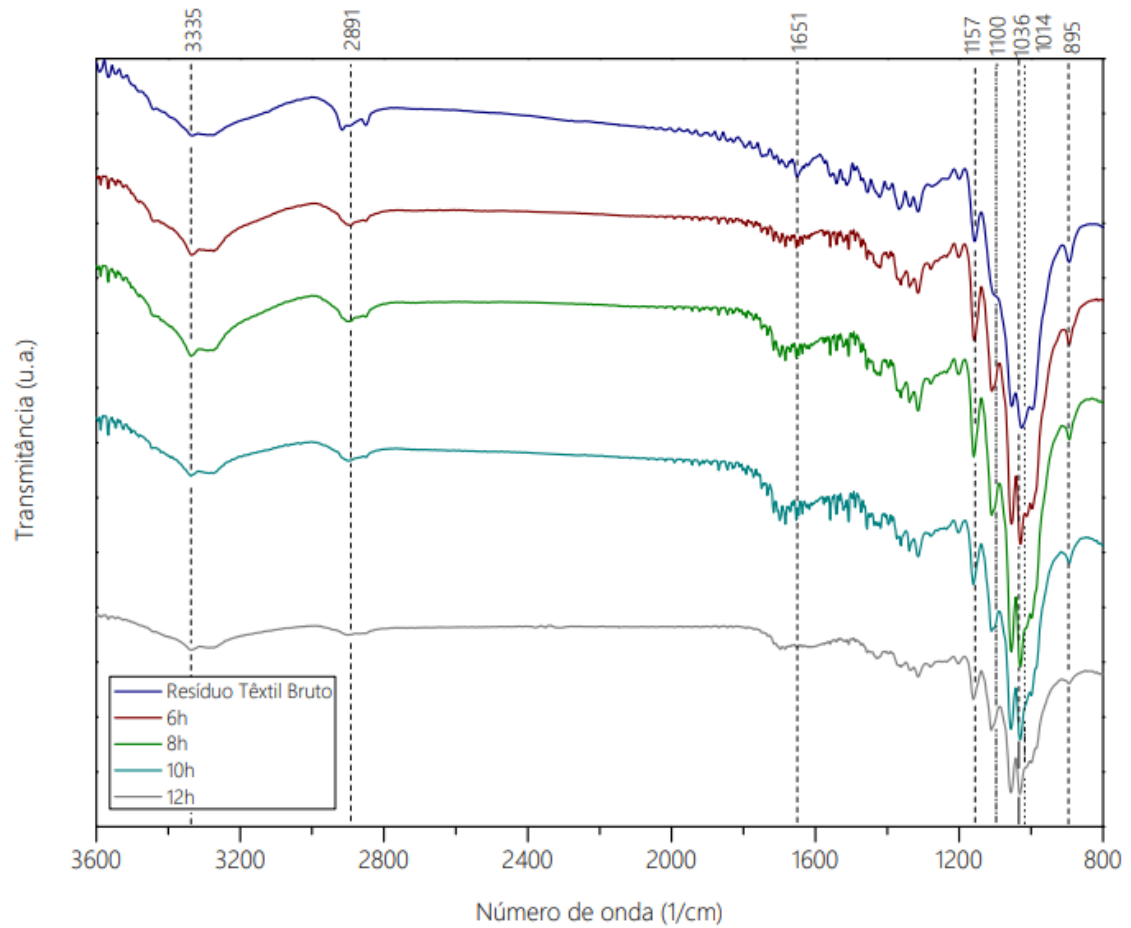

Figura 3: FTIR-ATR do resíduo têxtil bruto e seus hidrocarvões.

A fibra de algodão é constituída em cerca de 90 a $93 \%$ de celulose, sendo a maior parte restante constituída por ceras, gorduras ou minerais, etc.

Na Figura 3 podem-se observar diversas bandas características da celulose. A banda $3335 \mathrm{~cm}^{-1}$ pode ser atribuída ao estiramento $v(\mathrm{O}-\mathrm{H})$ e a banda em $2891 \mathrm{~cm}^{-1}$ pode ser atribuída aos estiramentos simétricos e antissimétricos de $v(\mathrm{C}-\mathrm{H})$ alifático e $v\left(\mathrm{CH}_{2}\right)$ alifático. A banda em $1651 \mathrm{~cm}^{-1}$ pode ser atribuída à deformação $\delta(\mathrm{O}-\mathrm{H})$. Em $1157 \mathrm{~cm}^{-1}$ podemos observar estiramento antissimétrico de $v(\mathrm{C}-\mathrm{O}-\mathrm{C})$ em ligação 
$\beta$-glucosídica. A banda em $1100 \mathrm{~cm}^{-1}$ pode ser atribuída ao estiramento $v(\mathrm{O}-\mathrm{H})$ antissimétrico do anel glicosídico. Em $1036 \mathrm{~cm}^{-1}$ observa-se deformação $(\mathrm{C}-\mathrm{O})$ de álcoois primários, em $1014 \mathrm{~cm}^{-1}$ vibração da ligação $(\mathrm{C}-\mathrm{O}-\mathrm{C}) \beta-1,4$, em $895 \mathrm{~cm}^{-1}$ grupos $-\mathrm{C}$ anoméricos e deformação $\delta(\mathrm{C}-\mathrm{H})[41]$.

Observa-se por meio da Figura 3, que as bandas em torno de 2800 e $2900 \mathrm{~cm}^{-1}$ diminuem de intensidade com o aumento do tempo de hidrocarbonização, implicando na perda do grupo $-\mathrm{CH}_{3}$ A diminuição de intensidade dessa banda representa o mecanismo de desmetilação durante a reação de CHT [41]. O mesmo está acontecendo com as bandas atribuídas ao estiramento $\mathrm{OH}$, a redução deste pico ocorre devido à reação de desidratação durante a CHT [6], que é a principal reação na formação dos hidrocarvão.

As ligações glucosídicas entre carbono e oxigênio da celulose, absorvem na mesma região de compostos oxidados de carbono, como carbonatos [6]. Essas ligações carbono-oxigênio podem estar ocorrendo durante a formação dos hidrocarvões. Por essa razão, as bandas relacionadas às ligações glicosídicas não apresentaram alteração.

Também foi investigado o DRX dos hidrocarvões de 6,8,10 e 12 horas. As estruturas cristalinas do material de carbono foram confirmadas pelo DRX, conforme mostra a Figura 4.

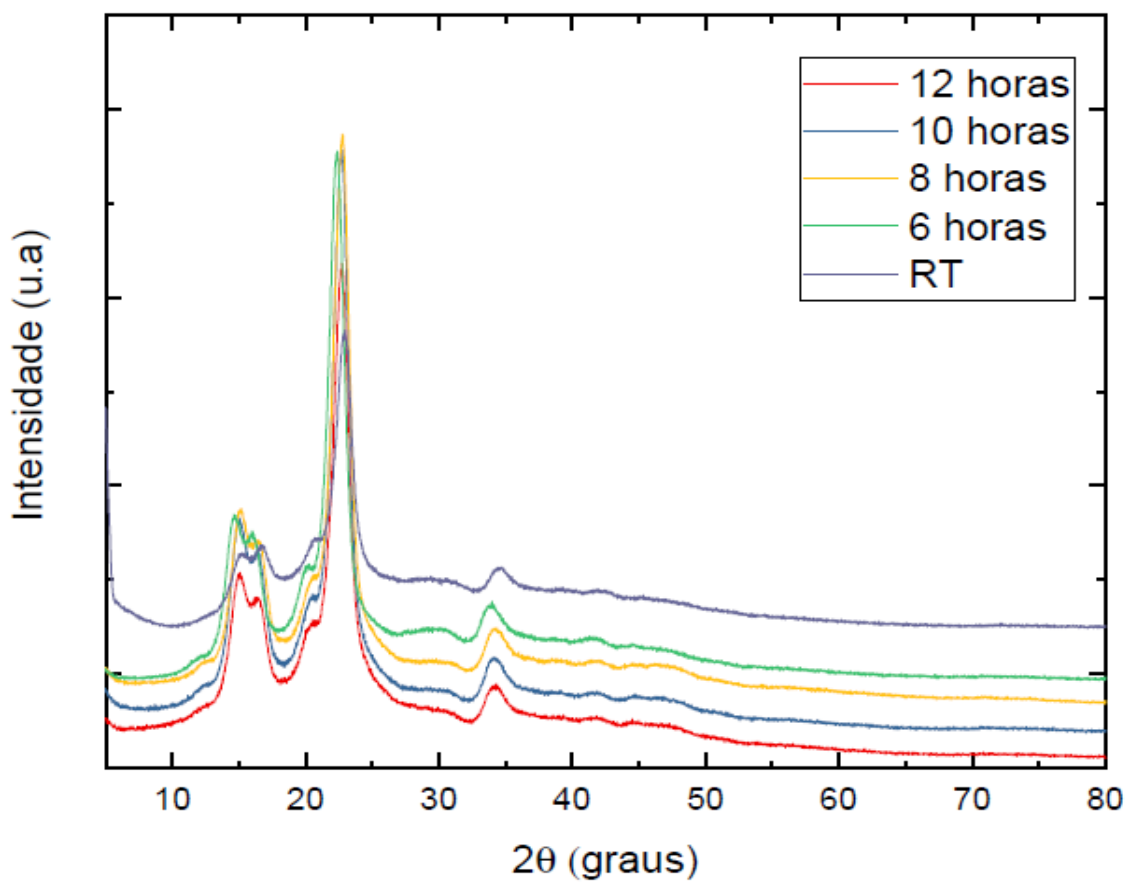

Figura 4: DRX das amostras de hidrocarvões e do resíduo têxtil bruto.

O pico de difração em $16^{\circ}, 22,7^{\circ}$ e $34,6^{\circ}$ são picos característicos da estrutura de celulose [42].

No presente estudo, realizado em temperatura de $220^{\circ} \mathrm{C}$, os picos cristalinos foram claramente observados em todos os tempos de hidrocarbonização, tendo alteração somente na intensidade destes picos. Os resultados indicam que a estrutura cristalina foi preservada, corroborando com os resultados obtidos por FTIR-ATR, (Figura 3). Resultados semelhantes foram reportados por NAKASON; PANYAPINYOPOL; KANOKKANTAPONG [43], que produziram hidrocarvão de rizoma de mandioca utilizando temperatura de $\left(160-200{ }^{\circ} \mathrm{C}\right)$. Pode se considerar que o processo de hidrocarbonização esteja ocorrendo superficialmente com o avanço moderado das reações. Apesar da análise de DRX sugerir que não houve modificação no material após a carbonização, as demais caracterizações demonstram que o material carbonizou não completamente. Assim, o hidrocarvão com o tempo de carbonização de 12 horas (HC12) foi o selecionado para prosseguir os experimentos.

O ponto de carga zero $\left(\mathrm{pH}_{\mathrm{pcz}}\right)$ pode ser visto na Figura 5. O HC12 teve um $\mathrm{pH}_{\mathrm{pcz}}$ de 3,35, ou seja, em soluções de $\mathrm{pH}$ acima desse valor está carregado negativamente, o que está de acordo com resultados prévios $[12,13]$. Assim sendo, a adsorção de compostos catiônicos, como o azul de metileno, é favorecida. 


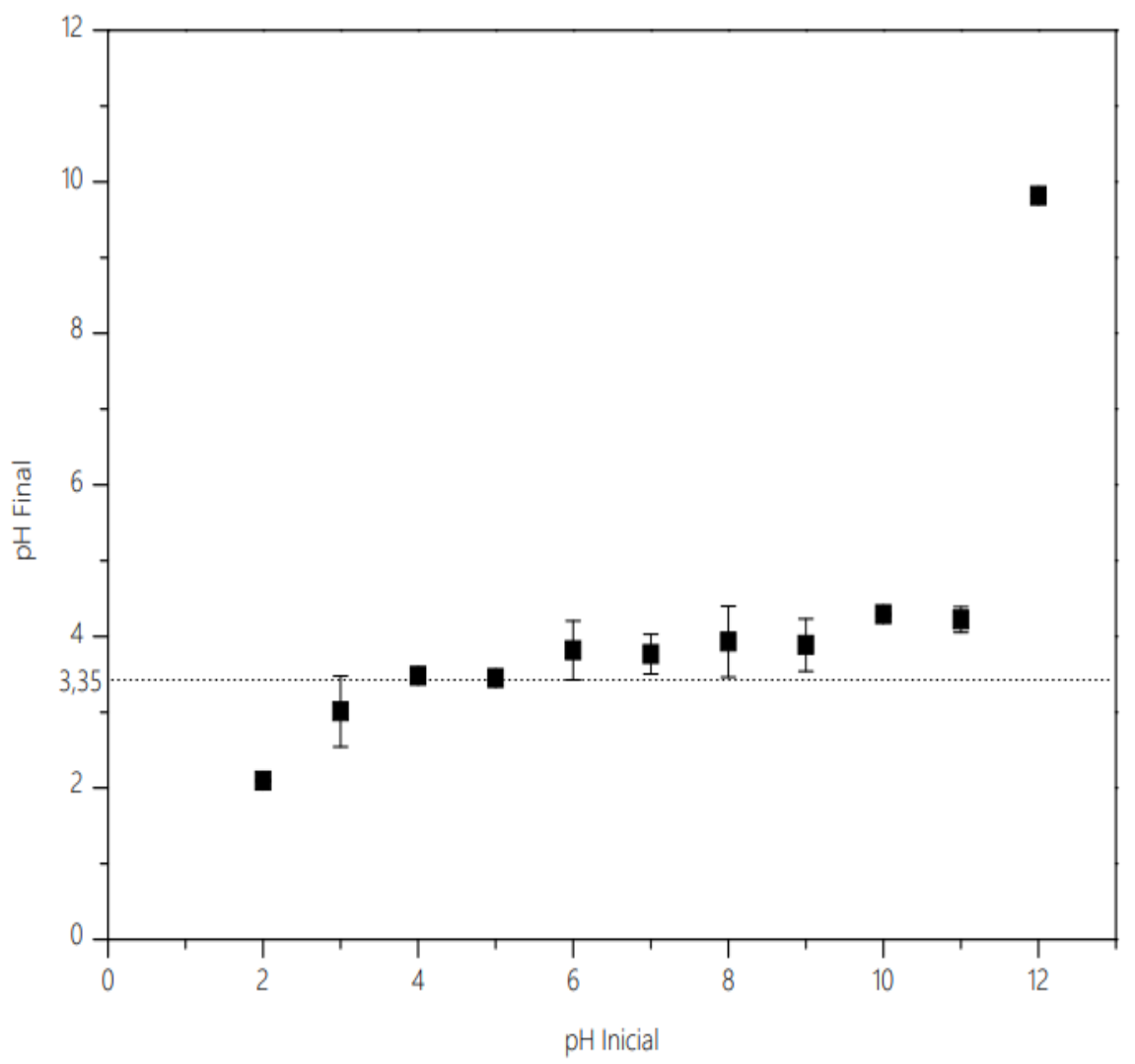

Figura 5: Ponto de carga zero do $\mathrm{HC} 12$.

\subsection{Estudo de adsorção de azul de metileno}

Inicialmente foi verificada a influência do pH da solução na adsorção de azul de metileno no HC12, pois o $\mathrm{pH}$ pode causar alterações nas cargas de superfície, influenciando o grau de protonação de grupos funcionais na superfície do adsorvente [44].

Na Figura 6 a adsorção de azul de metileno no $\mathrm{HC} 12$ foi realizada em diferentes valores de $\mathrm{pH}$. Verificou-se que, como esperado, com o aumento do $\mathrm{pH}$ aumenta-se a capacidade de adsorção do azul de metileno em hidrocarvão. $\mathrm{O}$ valor de adsorção em meio ácido é baixo quando comparado com a adsorção em meio básico. Em pH de solução igual a 3 o hidrocarvão adsorve apenas $36,30 \mathrm{mg} \cdot \mathrm{g}^{-1} \mathrm{e} \mathrm{em} \mathrm{pH} 10$, adsorve 65,59 $\mathrm{mg} \cdot \mathrm{g}^{-1}$. A baixa quantidade de corante adsorvida em $\mathrm{pH}$ baixo está associada a repulsões eletrostáticas entre as moléculas de azul de metileno (natureza catiônica) e os íons $\mathrm{H}^{+}$presentes na superfície do $\mathrm{HC} 12$ [45]. O aumento da adsorção em $\mathrm{pH}$ elevado pode ser atribuído à desprotonação de abundantes grupos carboxílicos ($\mathrm{COOH}-)$ na superfície, gerando interações eletrostáticas entre as moléculas de corante carregadas positivamente e a superfície negativamente carregada do adsorvente [46].

Resultados semelhantes foram obtidos por SEWU et al. [47]e SUN et al. [48], que mostraram que a adsorção de corantes catiônicos foi eficiente em $\mathrm{pH}$ alto. Para os estudos de adsorção subsequentes o pH da solução foi ajustado para pH 10. 


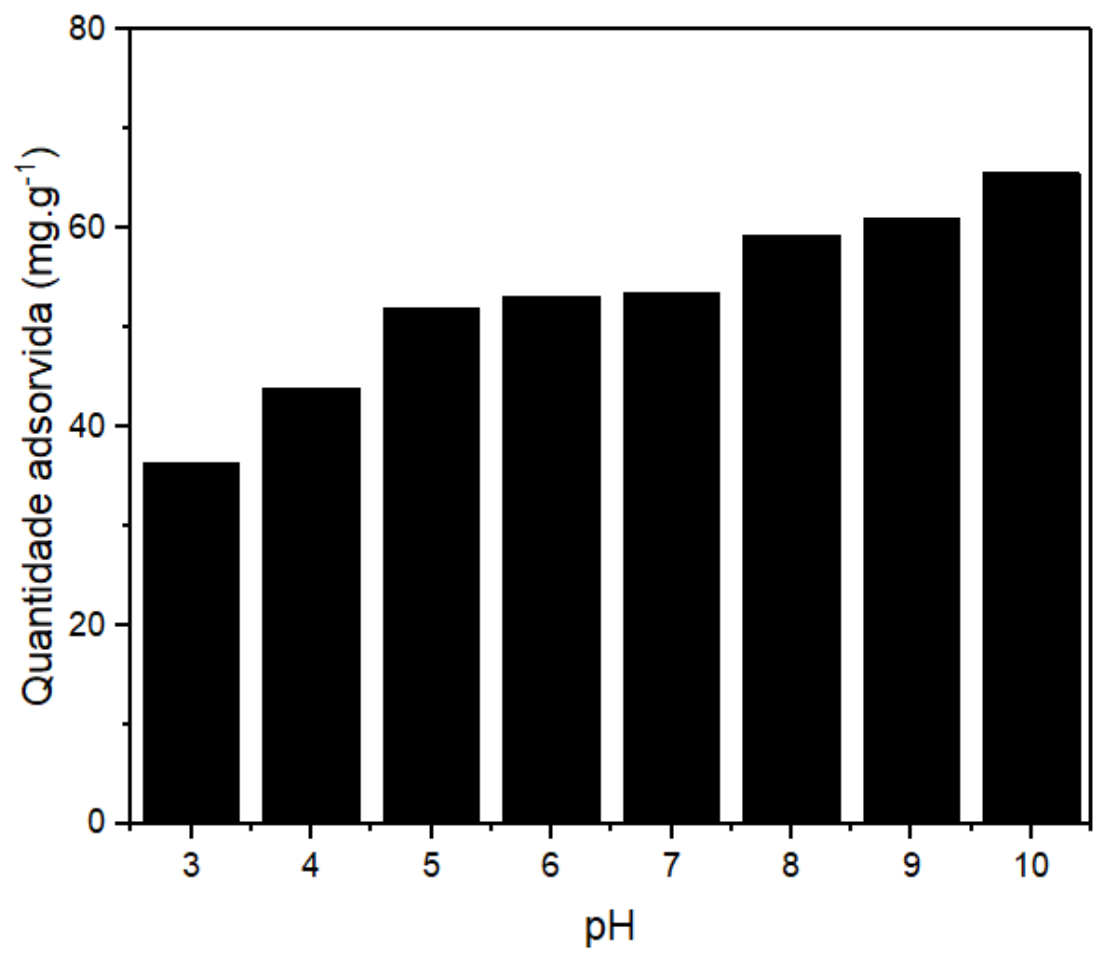

Figura 6: Efeito de pH da solução na adsorção do azul de metileno no HC12.

\subsubsection{Cinética de adsorção}

As quantidades de azul de metileno adsorvidos foram investigadas em função do tempo entre 0 e 120 min, para determinar o tempo de equilíbrio do processo de adsorção, como mostrado na Figura 7, onde também se observa os ajustes aos modelos cinéticos.

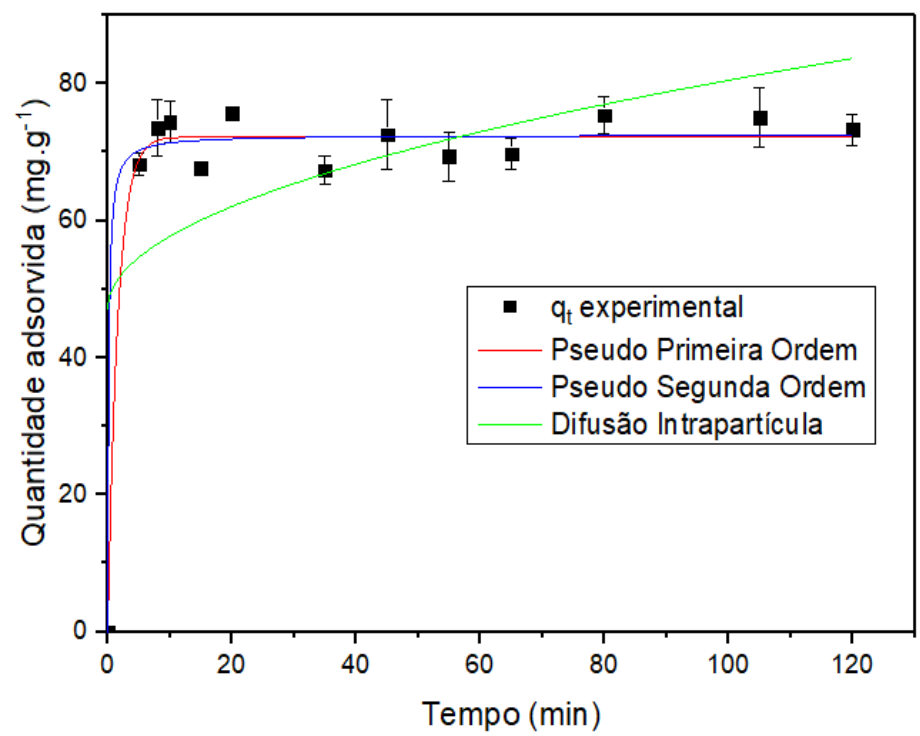

Figura 7: Cinética de adsorção do azul de metileno no HC12.

A adsorção do azul de metileno é muito rápida, atingindo o equilíbrio próximo a 7 minutos, o que indica uma boa afinidade do adsorvente pelo adsorvato. Esse comportamento se deve ao fato de que, há uma grande quantidade de sítios vazios para a adsorção [49]. Observa-se que a máxima quantidade retida foi de 
72,6 mg.g ${ }^{-1}$, que é um valor interessante para processos adsortivos, quando comparado com carvões não comerciais.

Observa-se na Figura 7 que o modelo pseudo segunda-ordem foi o que melhor se ajustou aos dados experimentais. Esse fato pode ser comprovado na Tabela 3, que lista os parâmetros dos ajustes cinéticos.

Tabela 3: Parâmetros dos modelos de cinética ajustados aos dados experimentais.

\begin{tabular}{c|c|c}
\hline Pseudo Primeira-Ordem & Pseudo Segunda-Ordem & Difusão Intrapartícula \\
\hline $\mathrm{q}_{\mathrm{eq}}=72,22 \pm 1,260$ & $\mathrm{q}_{\mathrm{eq}}=72,51 \pm 0,938$ & $\mathrm{C}=47,18 \pm 10,073$ \\
\hline $\mathrm{k}_{1}=0,61601 \pm 0,189$ & $\mathrm{k}_{2}=0,085961 \pm 0,124$ & $\mathrm{k}_{3}=3,3320 \pm 1,53$ \\
\hline $\mathrm{R}^{2}=0,9777$ & $\mathrm{R}^{2}=0,9762$ & $\mathrm{R}^{2}=0,2376$ \\
\hline$X^{2}=9,6913$ & $X^{2}=9,0784$ & $X^{2}=310,19$ \\
\hline
\end{tabular}

$\mathrm{q}_{\mathrm{eq}}=\mathrm{mg} \cdot \mathrm{g}^{-1} ; \mathrm{k}_{1}=\min ^{-1} ; \mathrm{k}_{2}=\mathrm{g} \cdot \mathrm{mg}^{-1} \cdot \min ^{-1} ; \mathrm{C}=\mathrm{mg} \cdot \mathrm{g}^{-1} ; \mathrm{k}_{3}=\mathrm{mg} \cdot \mathrm{g}^{-1} \cdot \mathrm{min}^{-1 / 2}$.

Observa-se que o modelo de pseudo segunda-ordem obteve um elevado valor para $\mathrm{R}^{2}$, associado ao mais baixo valor para $X^{2}(9,0784)$. O modelo de pseudo segunda-ordem foi, portanto, eleito como aquele que representa os dados cinéticos de remoção de azul de metileno na amostra HC12.

O modelo pseudo segunda-ordem indica que o processo de adsorção é significativamente controlado por quimissorção, que envolveu forças de valência por meio de compartilhamento ou troca de elétrons entre adsorvente e adsorvato [50, 51].

\subsubsection{Isoterma de adsorção}

A Figura 8 apresenta os dados experimentais bem como os ajustes dos modelos de isoterma. Observase que a isoterma é bastante favorável, o que corrobora com os dados cinéticos. O platô é bastante pronunciado e a capacidade máxima de adsorção do azul de metileno no HC12 foi de $140,35 \mathrm{mg} \cdot \mathrm{g}^{-1}$. A atração dos sítios do adsorvente, carregados negativamente por conta do $\mathrm{pH}$ da solução contribuíram para este comportamento.

Os modelos de Langmuir, Freundlich e Sips foram ajustados aos dados experimentais da isoterma de adsorção do azul de metileno e os parâmetros encontrados pelos modelos são apresentados na Tabela 4.

Tabela 4: Parâmetros dos modelos de isoterma ajustados aos dados experimentais.

\begin{tabular}{c|c|c}
\hline Langmuir & Freundlich & Sips \\
\hline $\mathrm{q}_{\max }=141,78_{ \pm} 4,233$ & $\mathrm{k}=76,982_{ \pm} 8,200$ & $\mathrm{q}_{\max }=141,61 \pm 5,086$ \\
\hline $\mathrm{b}=1,8293_{ \pm} 0,3026$ & $\mathrm{n}=7,3006_{ \pm} 1,403$ & $\mathrm{~b}=1,8407_{ \pm} 0,3524$ \\
\hline $\mathrm{R}^{2}=0,9409$ & $\mathrm{R}^{2}=0,7574$ & $\mathrm{R}^{2}=0,9350$ \\
\hline$X^{2}=120,523$ & $X^{2}=494,756$ & $X^{2}=132,544$ \\
$\mathrm{q}_{\max }=\mathrm{mg} \cdot \mathrm{g}^{-1} ; \mathrm{b}=\mathrm{L} \cdot \mathrm{mg}^{-1} ; \mathrm{k}=\mathrm{L} \cdot \mathrm{g}^{-1}$. & &
\end{tabular}

Por meio da Tabela 4, é possível averiguar que o modelo de Langmuir se ajustou aos dados experimentais da isoterma de adsorção do azul de metileno no hidrocarvão HC12, pois o coeficiente de correlação foi maior neste modelo e o $X^{2}$ foi menor. Os erros associados aos parâmetros também são pequenos comparados aos dos outros ajustes. O modelo de Langmuir, que prevê a existência de um platô pronunciado, consequência de significativa atração adsorvente/adsorvato, consegue, portanto, se adequar melhor aos dados de equilíbrio que expressam a forte atração por cargas opostas entre o sólido e o corante em pH 10. De fato, a capacidade máxima teórica da adsorção do azul de metileno no $\mathrm{HC} 12$ foi de $141,78 \mathrm{mg} \cdot \mathrm{g}^{-1}$, bem próximo do 
experimental. Além disso, o valor da constante de Langmuir, $b$, é bastante elevado, consequência exatamente da forte atração já mencionada.

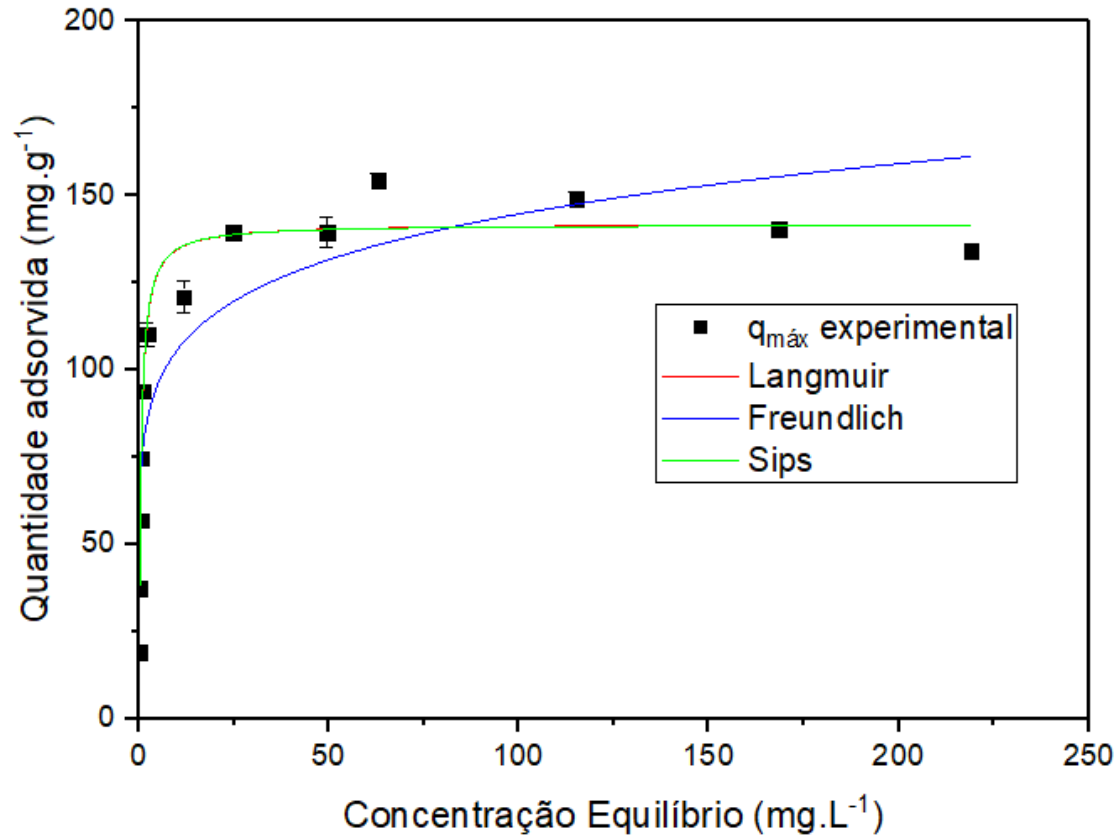

Figura 8: Modelos de isoterma ajustados aos dados experimentais.

Apesar do modelo de Freundlich não ter se ajustado tão bem quanto o de Langmuir, é possível obter conclusões da isoterma com o auxílio dele. O parâmetro empírico $n$ é usado como um índice de medição quanto ao grau de favorabilidade do processo de adsorção, em que a adsorção é favorável quando $n>1$ [52]. Assim, neste estudo em que o $\mathrm{n}=7,301$ é um indicativo, além da própria forma da isoterma, que a adsorção de azul de metileno no HC12 é favorável.

O modelo de Sips, que engloba parâmetros dos modelos anteriores também se ajusta bem aos dados experimentais. Porém, dá-se preferência para o modelo de dois parâmetros, mais simples, o de Langmuir.

Para efeito comparativo, é apresentado na Tabela 5 os resultados de capacidade máxima adsorvida de azul de metileno em diferentes materiais.

Tabela 4: Máxima capacidade de adsorção do azul de metileno em diversos materiais. 


\begin{tabular}{c|c|c}
\hline ADSORVENTE & $\begin{array}{c}\text { QUANTIDADE } \\
\text { ADSORVIDA MG.G }{ }^{-1}\end{array}$ & REFERÊNCIA \\
\hline Hidrocarvão de resíduo têxtil & 140,35 & Este trabalho \\
\hline Carvão ativado comercial & 48,3 & (LONAPPAN et al., 2016) \\
\hline $\begin{array}{c}\text { Hidrocarvão ativado pelo método tradicional com } \\
\text { NaOH de casca de coco }\end{array}$ & 107,0 & (ISLAM et al., 2017b) \\
\hline Espiga do milho & 80,7 & (MIRABOUTALEBI et al., \\
\hline Hidrocarvão sem ativação de casca de café & 34,9 & (RONIX et al., 2017) \\
\hline Hidrocarvão de lama vermelha & 76,92 & (SALIMI et al., 2017) \\
\hline Kidrocarvão ativado pelo método tradicional com & 93,4 & (NOGUEIRA, DUARTE, \\
\hline Hidrocarvão sem ativação de resíduos de acerola & 108,88 & BARROZO, 2019)
\end{tabular}

É notável que a capacidade de adsorção do $\mathrm{HC} 12$ é superior aos demais hidrocarvões, carvões e biomassas. Logo, todas as evidências levam a concluir, que o HC12 é um excelente adsorvente do corante em questão. De acordo com os dados apresentados, é possível avaliar que o hidrocarvão de resíduos têxteis tem um grande potencial a ser explorado no tratamento de efluentes têxteis.

\section{CONCLUSÃO}

A realização deste trabalho permitiu que fossem avaliados os parâmetros da CHT de resíduos de tecido de algodão de forma a encontrar uma condição favorável para a produção do hidrocarvão com alta concentração de carbono. Por fim, foi avaliada a capacidade de adsorção do hidrocarvão selecionado. Observou-se que o hidrocarvão produzido em 12 horas é o que apresenta as melhores características de carvão, com 71,2\% de carbono na sua composição.

O hidrocarvão produzido a partir de resíduos têxteis sem ativação foi satisfatoriamente aplicado na adsorção de azul de metileno em meio aquoso.

Ao ser investigado, o $\mathrm{HC} 12$ teve um $\mathrm{pH}_{\mathrm{pcz}}$ de 3,35 indicando características ácidas da superfície. Estudos de adsorção mostraram um aumento na capacidade de adsorção do azul de metileno à medida que o pH da solução foi aumentado. Os dados cinéticos experimentais se ajustaram melhor ao modelo de pseudo segunda-ordem sugerindo o compartilhamento ou troca de elétrons entre adsorvente e adsorvato. A isoterma de adsorção mostrou que o hidrocarvão sem ativação tem grande capacidade adsortiva do azul de metileno, de $140,35 \mathrm{mg} \cdot \mathrm{g}^{-1}$ semelhante à capacidade de carvões ativados. E o modelo de isoterma que melhor se ajustou foi o de Langmuir, sugerindo que a adsorção ocorre em monocamada. Em vista disso, é possível concluir que o método de hidrocarbonização é viável no tratamento de efluentes líquidos poluídos com corante catiônico, como o azul de metileno.

\section{BIBLIOGRAFIA}

[1] SANDIN, G.; PETERS, G. M. "Environmental impact of textile reuse and recycling e A review". Journal of Cleaner Production, v. 184, pp. 353-365, 2018.

[2] MENEGUCCI, F.; MARTELI, L. Resíduos têxteis : Análise sobre descarte e reaproveitamento nas indústrias de confecção. n. August, 2015.

[3] ROMANO, M. S., et al. "Novel carbon materials for thermal energy harvesting". Journal of Thermal Analysis and Calorimetry, v. 109, pp. 1229-1235, mar. 2012. 
[4] LIU, Y.; MA, S.; CHEN, J. "A novel pyro-hydrochar via sequential carbonization of biomass waste : Preparation, characterization and adsorption capacity". Journal of Cleaner Production, v. 176, pp. 187-195, 2018.

[5] ZHANG, L., et al. Hydrothermal Carbonization of Corncob Residues for Hydrochar Production. 2014.

[6] LIN, Y., et al. "A Mechanism Study on Hydrothermal Carbonization of Waste Textile". Energy and Fuels, v. 30, n. 9, pp. 7746-7754, 2016.

[7] ZHAO, P., et al. "Clean solid biofuel production from high moisture content waste biomass employing hydrothermal treatment". APPLIED ENERGY, v. 131, pp. 345-367, 2014.

[8] AREEPRASERT, C., et al. Alternative Solid Fuel Production from Paper Sludge Employing Hydrothermal Treatment. 2014.

[9] KIM, D.; LEE, K.; PARK, K.Y. "Hydrothermal carbonization of anaerobically digested sludge for solid fuel production and energy recovery". FUEL, v. 130, pp. 120-125, 2014

[10] FALCO, C., et al. "Hydrothermal carbon from biomass: Structural differences between hydrothermal and pyrolyzed carbons via 13C solid state NMR". Langmuir, v. 27, pp. 14460-14471, 2011.

[11] ALMEIDA, C., et al. "Transforming Sugarcane Bagasse and Vinasse Wastes into Hydrochar in the Presence of Phosphoric Acid : An Evaluation of Nutrient Contents and Structural Properties". Waste and Biomass Valorization, v. 8, n. 4, pp. 1139-1151, 2017.

[12] GUILHERME, M.R., et al. "Hydrochars based on cigarette butts as a recycled material for the adsorption of pollutants". Biochemical Pharmacology, 2018.https://doi.org/10.1016/j.jece.2018.11.012 acessado em setembro de 2019.

[13] KUPFER, V. L., et al. PT. "Synthesis and characterization of pecan nutshell-based adsorbent with high specific area and high methylene blue adsorption capacity". Journal of Molecular Liquids, https://doi.org/10.1016/j.molliq.2018.12.010 acessado em setembro de 2019, v. 276, pp. 570-576, 2018.

[14] LANG, Q., et al. "Properties of hydrochars derived from swine manure by $\mathrm{CaO}$ assisted hydrothermal carbonization". Journal of Environmental Management, v. 233, n. June 2018, pp. 440-446, 2019.

[15] LI, L., et al. "Hydrothermal carbonization of food waste and associated packaging materials for energy source generation". Waste Management, v. 33, n. 11, pp. 2478-2492, 2013.

[16] PRAWISUDHA, P.; NAMIOKA, T.; YOSHIKAWA, K. "Coal alternative fuel production from municipal solid wastes employing hydrothermal treatment". Applied Energy, v. 90, n. 1, pp. 298-304, 2012.

[17] ZHU, G., et al. "Characterization and pelletization of cotton stalk hydrochar from HTC and combustion kinetics of hydrochar pellets by TGA”. Fuel, v. 244, n. November 2018, pp. 479-491, 2019.

[18] MITROPOULOS, A.C. "Chemical Engineering Research and Design Graphene composites as dye adsorbents : Review". Chemical Engineering Research and Design, v. 129, pp. 75-88, 2017

[19] DI BERNARDO, L., DANTAS, A. Métodos e Técnicas de Tratamento de Água. 2a ed ed. São Carlos, SP: RIMA, 2005

[20] PELEKANI, C., SNOEYINK, V. L. Competitive adsorption between atrazine and methylene blue on activated carbon : the importance of pore size distribution. v. 38, pp. 1423-1436, 2000

[21] ABNT. NBR. 13538:1995. Análise qualitativa. Rio de Janeiro, nov. 1995

[22] ABNT. NBR. 11914:1992.Análise quantitativa de materiais têxteis. Rio de Janeiro, abr. 1992.

[23] PERES, E. C., et al. "Microwave synthesis of silica nanoparticles and its application for methylene blue adsorption". Journal of Environmental Chemical Engineering, v. 6, pp. 649-659, 2018

[24] FAGNANI, H.M.C., et al. "CO 2 adsorption in hydrochar produced from waste biomass". SN Applied Sciences, v. 1, n. 9, pp. 1-10, 2019.

[25] PARK, J.; REGALBUTO, J.R. "A simple, accurate determinatio of oxide PZC and the strong buffering effect os oxide surfaces at incipient wetness". Journal of Colloid and Interface Science, v. 175, pp. 239-252, 1995.

[26] HO, Y.S.; MCKAY, G. "Pseudo-second order model for sorption processes". Process Biochemistry, v. 34, p. 451-465, jul. 1999.

[27] LAGERGREN, S. "Zur theorie der sogenannten adsorption geloster stoffe. Kungliga svenska vetenskapsakademiens". Handlingar, v. 24, pp. 1-39, 1898. 
[28] WEBER, W.J.; MORRIS, J.C. "Kinetics of adsorption on carbon from solution". Journal Sanitary Engeering Division Proceedings, v. 89, pp. 31-59, 1963.

[29] LANGMUIR, I. "The adsorption of gases on plane surfaces of glass, mica and platinum". Journal of American Chemical Society, v. 40, pp. 1361-1403, 1918.

[30] FREUNDLICH, H.M.F. “Über die adsorption in lösungen”. Zeitschrift für Physikalische Chemie, v. 57, pp. 385-470, 1906.

[31] SIPS, R. "Combined form of Langmuir and Freundlich equations". The Journal of Chemical Physics, v. 16, pp. 490-495, 1948.

[32] BASSO, D., et al. "Agro-industrial waste to solid biofuel through hydrothermal carbonization". WASTE MANAGEMENT, 2015.

[33] CHEN, X., et al. "Conversion of sweet potato waste to solid fuel via hydrothermal carbonization". Bioresource Technology, 2017.

[34] GUO, S., et al. "carbonization of corn stalk". Journal of Analytical and Applied Pyrolysis, 2015.

[35] ROMÁN, S., et al. "Hydrothermal carbonization as an effective way of densifying the energy content of biomass". Fuel Processing Technology, v. 103, pp. 78-83, nov. 2012.

[36] TEKIN, K.; AKALIN, M.K. The effects of water tolerant Lewis acids on the hydrothermal liquefaction of lignocellulosic biomass. pp. 1-9, 2015.

[37] VOLPE, M.; GOLDFARB, J.L.; FIORI, L. "Hydrothermal carbonization of Opuntia ficus-indica cladodes: Role of process parameters on hydrochar properties". Bioresource Technology, v. 247, pp. 310-318, 2018.

[38] TITIRICI, M.M., et al. "Black perspectives for a green future: hydrothermal carbons for environment protection and energy storage”. Energy \& Environmental Science, v. 5, pp. 6796-6822, 2012.

[39] CAI, J., et al. "Bioresource Technology Hydrothermal carbonization of tobacco stalk for fuel application". Bioresource Technology, v. 220, pp. 305-311, 2016.

[40] SEVILLA, M.; MACIÁ-AGULLÓ, J.A.; FUERTES, A.B. "Hydrothermal carbonization of biomass as a route for the sequestration of $\mathrm{CO} 2$ : Chemical and structural properties of the carbonized products". Biomass and Bioenergy, v. 35, p. 3152-3159, jul. 2011.

[41] CASTRO, A.J.R. "Carbonização hidrotérmica de celulose e nanocelulose e preparação de compósitos magnéticos em uma única etapa". p. 94 (Tese de doutorado) - Universidade Federal do Ceará, 2018.

[42] ZHANG, L., et al. "Effect of residence time on hydrothermal carbonization of corn cob residual". BioResources, v. 10, p. 3979-3986, 2015.

[43] NAKASON, K.; PANYAPINYOPOL, B.; KANOKKANTAPONG, V. "Characteristics of hydrochar and liquid fraction from hydrothermal carbonization of cassava rhizome". Journal of the Energy Institute, v. 91, n. 2, p. 184-193, 2018.

[44] SONG, W., et al. "High-capacity adsorption of dissolved hexavalent chromium using aminefunctionalized magnetic corn stalk composites". Bioresource Technology, v. 190, p. 550-557, 2015

[45] ISLAM, A., et al. "fruit hulls for methylene blue adsorption". Journal of the Taiwan Institute of Chemical Engineers, v. 0, p. 1-9, 2017a.

[46] QIAN, W., et al. "Ecotoxicology and Environmental Safety Removal of methylene blue from aqueous solution by modi fi ed bamboo hydrochar". Ecotoxicology and Environmental Safety, v. 157, n. February, p. 300-306, 2018.

[47] SEWU, D.D.; BOAKYE, P.; WOO, S.H. "Highly efficient adsorption of cationic dye by biochar produced with Korean cabbage waste". Bioresource Technology, 2016.

[48] SUN, P., et al. "Efficient removal of crystal violet using Fe 3 O 4 -coated biochar : the role of the Fe $3 \mathrm{O}$ 4 nanoparticles and modeling study their adsorption behavior". Nature Publishing Group, n. March, p. 1-12, 2015.

[49] MESTRE, A.S., et al. Activated carbons for the adsorption of ibuprofen. v. 45, p. 1979-1988, 2007.

[50] AKROUT, H.; JELLALI, S.; BOUSSELMI, L. "Enhancement of methylene blue removal by anodic oxidation using BDD electrode combined with adsorption onto sawdust". Comptes Rendus Chimie, v. 18, p. 110-120, 2015. 
[51] LI, Z., et al. "Methylene blue adsorption from aqueous solution by loofah sponge-based porous carbons". Colloids and Surfaces A: Physicochemical and Engineering Aspects, v. 538, p. 28-35, 2018.

[52] MCCABE, W.L.; SMITH, J.C.; HARRIOT, P. Unit operations of chemical engineering. $6^{\mathrm{a}}$ ed. [s.1.] McGraw Hill International, 2001.

\section{ORCID}

Adriana Ferreira de Lima

Helida Monique Cordasso Fagnani

https://orcid.org/0000-0001-5881-5905

Washington Luiz Félix Santo

https://orcid.org/0000-0002-9249-0603

Maria Angelica Simoes Dornellas de Barros

https://orcid.org/0000-0001-9351-143X

https://orcid.org/0000-0002-4088-4248 